\title{
Detection of microsatellite instability with Idylla MSI assay in colorectal and endometrial cancer
}

\author{
liris Ukkola ${ }^{1,2} \cdot$ Pirjo Nummela $^{2}$ (1) $\cdot$ Annukka Pasanen $^{1} \cdot$ Mia Kero ${ }^{1} \cdot$ Anna Lepistö ${ }^{2,3} \cdot$ Soili Kytölä $^{4} \cdot$ Ralf Bützow $^{1,2}$. \\ Ari Ristimäki ${ }^{1,2}$
}

Received: 8 January 2021 / Revised: 23 February 2021 / Accepted: 11 March 2021 / Published online: 23 March 2021

(C) The Author(s) 2021

\begin{abstract}
Universal testing of microsatellite instability (MSI) is recommended for colorectal cancer (CRC) and endometrial cancer (EC) to screen for Lynch syndrome and to aid in assessing prognosis and optimal treatment. We compared the performance of Idylla MSI test to immunohistochemistry (IHC) of mismatch repair (MMR) proteins in consecutive series of $100 \mathrm{CRC}$ and $108 \mathrm{EC}$ samples, as well as in retrospective series of $28 \mathrm{CRC}$ and $33 \mathrm{EC}$ specimens with known deficient MMR protein expression. The concordance between the Idylla test and IHC was $100 \%$ in all CRC samples $(n=128)$ but lower in EC samples $(87.2 \%$; $n=141$ ). In the EC samples, sensitivity of Idylla test was $72.7 \%$ and specificity $100 \%$. EC MSI/dMMR agreement was $85.4 \%$ for MLH1, 87.5\% for MSH2, and only 35.3\% for MSH6. When we analyzed 14 EC samples that were discrepant, i.e., dMMR using IHC and microsatellite stable using Idylla, with microsatellite markers BAT25 and BAT26, we found four cases to be replication error (RER) positive. All RER positive cases were deficient for MSH6 protein expression. We also re-analyzed EC samples with variable tumor cellularity to determine the limit of detection of the Idylla test and found that a $30 \%$ or higher tumor cellularity is required. We conclude that Idylla MSI test offers a sensitive and specific method for CRC diagnostics but is less sensitive in EC samples especially in the case of MSH6 deficiency.
\end{abstract}

Keywords Colorectal cancer $\cdot$ Endometrial cancer $\cdot$ Idylla $\cdot$ Immunohistochemistry $\cdot$ Microsatellite instability $\cdot$ Mismatch repair

\section{Introduction}

Microsatellites are short repetitive DNA sequences that are prone to replication errors (RER). Microsatellite instability (MSI) is caused by deficient mismatch repair (dMMR) system, leading to hypermutation phenomenon and cancer susceptibility $[1,2]$. Approximately $15 \%$ of colorectal cancers

Ari Ristimäki

ari.ristimaki@helsinki.fi

1 Department of Pathology, HUSLAB, HUS Diagnostic Center, Helsinki University Hospital and University of Helsinki, P.O. Box 400, HUS, FI-00029 Helsinki, Finland

2 Applied Tumor Genomics Research Program, Research Programs Unit, University of Helsinki, Helsinki, Finland

3 Department of Gastrointestinal Surgery, Helsinki University Hospital, Helsinki, Finland

4 Department of Genetics, HUSLAB, HUS Diagnostic Center, Helsinki University Hospital, Helsinki, Finland
(CRC) and $30 \%$ of endometrial cancers (EC) arise from MSI pathway [3]. Most MSI cancers are sporadic and account for approximately $90 \%$ of the MSI EC cases and $80 \%$ of the MSI CRC cases, which most often develops through acquired MLH1 promoter hypermethylation [4, 5]. Lynch syndrome (LS), on the other hand, is a hereditary form of MSI most often caused by germline mutation in one of the MMR genes (MLH1, MSH2, MSH6, or PMS2) [2]. To this end, universal testing of MSI is recommended for CRC and EC patients to screen for LS and to aid in assessing prognosis and determining optimal treatment and follow-up [6-8].

Immunohistochemistry (IHC) technique is used in many pathology laboratories as a standard method to detect the loss of MMR protein expression to screen dMMR cases. The other standard method is PCR-based microsatellite test that consists of variable number and kinds of microsatellite markers, including at least mononucleotide markers BAT25 and BAT26, and is an alternative method for IHC MMR testing, especially in case of doubtful IHC results. [6] The sensitivity to detect MSI in dMMR CRC tumors using PCR-based microsatellite tests has been 
reported to be $89 \%$ for MLH1/MSH2 deficient but only $77 \%$ for MSH6 deficient cases [9], and in EC, the sensitivity has been estimated to range from 41 to $100 \%$ and the specificity from 69 to $89 \%$ [10].

The requirement of extensive hands-on time and trained personnel for above-mentioned IHC and PCR analysis, along with increasing demand of MSI testing, makes fast and automated molecular methods attractive alternatives. One novel way to assess the MSI status is Idylla MSI test, which analyzes a panel of seven monomorphic microsatellite biomarkers (ACVR2A, BTBD7, DIDO1, MRE11, RYR3, SEC31A, and $S U L F 2$ ) using fluorescent-labeled molecular beacons combined with PCR amplification. Idylla test is an automatic system performing all the necessary steps from a formalin-fixed paraffin-embedded (FFPE) tissue flake to MSI status information, including DNA extraction, amplification, and data analysis in $150 \mathrm{~min}$ [11].

The aim of this study was to evaluate the diagnostic performance of the Idylla MSI test as compared to the routine MMR IHC in CRC and EC samples. Analysis was performed in both consecutive sample series (CRC $n=100$ and EC $n=108$ ) and in retrospective series (CRC $n=28$ and EC $n=33$ ) with known dMMR IHC result. In addition, we scrutinized the minimum tumor cellularity requirement for the Idylla MSI test to detect the MSI status in the EC samples.

\section{Materials and methods}

\section{Sample selection}

We analyzed a prospective and consecutive series of $100 \mathrm{CRC}$ samples (CRC Set I, of which one was an appendix adenocarcinoma and one a colon descendens adenoma) from patients who underwent surgical resection at Helsinki University Hospital (HUH) between February and April 2019. These patients were routinely screened for MMR proteins MLH1, MSH2, MSH6, and PMS2 in real-life diagnostic setting using IHC and experimentally tested in blinded manner for MSI using Idylla MSI test at the Meilahti Pathology Department, Helsinki, Finland. We also collected a consecutive series of 108 EC samples (EC Set I) from patients operated at HUH between February 2018 and March 2020, which were analyzed in blinded manner using Idylla MSI test. In addition to the consecutive EC Set I, we collected a historical EC series (EC Set II; $n=33$ ) with known dMMR protein IHC result from patients operated at HUH between January 2007 and February 2012 and reanalyzed the samples using IHC and Idylla. A retrospective CRC Set II consisted of 28 samples with known IHC result of dMMR for MSH2, MSH6, or PMS2 and operated at HUH between October 2017 and September 2020, which had not been included to the CRC Set I. This study was approved by the Ethics Committee of the Helsinki University Central Hospital.

\section{Immunohistochemistry}

IHC was done on all CRC and EC tissue samples to detect the loss of MMR protein expression as a golden standard test using the following antibodies: MLH1 (clone ES05, diluted 1:50; Dako/Agilent, Santa Clara, CA), MSH2 (clone G2191129, diluted 1:400; BD Biosciences, San Jose, CA), MSH6 (clone EPR3945, diluted 1:200; Abgent, San Diego, CA), and PMS2 (clone EP51, diluted 1:50; Dako/Agilent, Santa Clara). The MSH2 and MSH6 stainings were performed with Ventana BenchMark ULTRA immunostainer (Roche, Ventana Medical Systems, Tucson, AZ, USA) utilizing OptiView DAB kit (760-700, Ventana/Roche). MLH1 and PMS2 stainings were performed with Autostainer (Agilent/ Dako, Santa Clara, USA) utilizing BrightVision detection kit (DPVB110HRP, Immunologic, WellMed, Duiven, the Netherlands). The loss of one or more MMR protein was defined as a dMMR, and the expression of all four MMR proteins was defined as proficient MMR (pMMR). Negative MMR protein expression was considered valid if nuclear staining in the tumor cells was absent with positive external (normal colon mucosa) and internal control staining (stromal nonneoplastic cells).

\section{Macrodissection}

The minimum tumor cell percentage instructed by the manufacturer for Idylla MSI test is $\geq 20 \%$ for CRC samples, and for the EC samples, we used $\geq 30 \%$ proportion of tumor cells. To increase the tumor cell percentage at or above the detection limit, macrodissection was performed for the FFPE tissue blocks of 4/100 CRC Set I samples, 71/108 EC Set I samples, 26/33 EC Set II samples, and 19/28 CRC Set II samples. After macrodissection, the tumor cell percentages were 20-90\% for CRC samples and 30-90\% for EC samples, as estimated from the HE slides by an experienced pathologist (AR). Manufacturer's protocol to perform the Idylla test requires a total tissue area between 25 and $300 \mathrm{~mm}^{2}$ with section thickness of $10 \mu \mathrm{m}$. For Idylla analysis, one or two $10-\mu \mathrm{m}$ tissue sections were cut from the FFPE tissue blocks with a Leica SM2000R microtome (Leica Microsystems GmbH, Wetzlar, Germany) using aseptic conditions.

\section{Idylla MSI test}

FFPE tissue samples were tested using automated Idylla MSI $^{\mathrm{TM}}$ Test (Biocartis NV, Mechelen, Belgium) that has been CE-IVD validated for CRC samples. The tissue handling and analysis were performed according to the manufacturer's protocol. The Idylla test result is considered 
valid if at least five out of the seven biomarkers (ACVR2A, BTBD7, DIDO1, MRE11, RYR3, SEC31A, and $S U L F 2$ ) are fully analyzed. The presence of at least two mutant biomarkers give rise to the judgment of MSI phenotype, whereas the presence of zero or one mutant biomarker indicates MSS phenotype.

\section{Determination of the limit of detection}

To scrutinize the limit of detection of the Idylla MSI test, we analyzed samples with variable tumor cell percentages. For that, we selected eight representative cases from the EC set I samples, which had been dMMR using IHC and MSI by the Idylla test. We then macrodissected these samples to achieve altogether 21 separate samples with tumor cellularity varying from 10 to $70 \%$, as estimated by AR, that were analyzed with the Idylla platform.

\section{Replication error status using BAT25 and BAT26 mismatch markers}

Discrepant samples between IHC and Idylla test were further analyzed with RER test using BAT25 and BAT26 mononucleotide repeats, in which $10-\mu \mathrm{m}$ FFPE tissue flakes were cut from whenever $50 \%$ or more tumor cells [12] could be retrieved and DNA was extracted from the deparaffinized flakes by using a Maxwell@ CSC Blood DNA Kit (Promega Corporation, Madison, WI) according to the manufacturer's instructions. MSI status was then assessed using the two mononucleotide-repeat markers using fluorescently labeled PCR. Detection of allelic variation in both BAT25 and BAT26 was considered as a positive result representing MSI.

\section{Statistical analysis}

The MMR IHC was considered as the golden standard reference test against which the overall agreement (concordance), sensitivity and specificity, and the positive predictive value and negative predictive value were calculated. To quantify the degree of agreement between IHC and the Idylla test, two-tailed Fisher's exact test was used (GraphPad QuickCalcs: https://www.graphpad.com/ quickcalcs/contingency2/). In order to compare the mutated biomarker spectrums between MSI CRC cases and MSI EC cases detected by the Idylla test, the unpaired $t$-test was used (GraphPad QuickCalcs: https:// www.graphpad.com/quickcalcs/ttest2/). $P$-value less than 0.05 was considered as statistically significant, and results are shown as means $\pm \mathrm{SD}$.

\section{Results}

\section{Comparison between Idylla MSI analysis and IHC in colorectal cancer}

One hundred consecutive CRC samples (CRC Set I) were analyzed in prospective, blinded, and real-life diagnostic setting by IHC of MMR proteins (MLH1, MSH2, MSH6, and PMS2) and Idylla MSI test. Of these patients, 44 were males and 56 females with a median age of 76 years (range from 36 to 96), and the tumors localized to the right colon $(n=54)$, the left colon $(n=33)$, and the rectum $(n=11)$. CRC tumor cell percentages estimated for the Idylla analysis varied between 20 and $90 \%$, and none of the Idylla test results were invalid. IHC and Idylla results showed a $100 \%$ agreement and were dMMR/MSI in 32 and $\mathrm{pMMR} / \mathrm{MSS}$ in 68 of the cases (Table 1; two-tailed Fisher's exact test, $P<0.0001)$. Based on IHC results, dMMR resulted from loss of MLH1 protein (and concomitant loss of PMS2 protein) expression in 31 cases and an isolated loss of MSH6 in one case.

Since sensitivity of MSI detection might depend on the type of MMR gene affected, we collected 28 retrospective CRC samples (Set II) with known dMMR IHC staining pattern. Based on IHC results, there was loss of MSH2 (and concomitant loss of MSH6) protein expression in 18, an isolated loss of MSH6 protein expression in 5, and an isolated loss of PMS2 protein expression in 5. CRC tumor cell percentages estimated for the Idylla analysis varied between 20 and $80 \%$, and none of the Idylla test results were invalid. Again, IHC and Idylla results showed a perfect $100 \%$ agreement. When the MSI cases of CRC Sets I and II were combined $(n=60)$, Idylla test detected three to seven mutated biomarkers in 59/60 samples and two mutated biomarkers in one sample, whereas all the MSS samples had seven wild-type biomarkers. On average, $82.1 \pm 12.9 \%$ of the seven unstable sites were mutated in the MSI CRC cases. Two markers, ACVR2A and $M R E 11$, were most commonly mutated (56 samples, 93.3\%), and SEC31A was least commonly mutated (35 samples, 58.3\%) (Fig. 1).

Table 1 Concordance between MMR protein IHC analysis and Idylla MSI test in the consecutive CRC Set I $(n=100)$

\begin{tabular}{llll}
\hline & & Idylla \\
\hline & & MSI & MSS \\
IHC & dMMR & 32 & 0 \\
& pMMR & 0 & 68 \\
\hline
\end{tabular}

$d M M R$, deficient MMR protein expression, MMR mismatch repair, MSI microsatellite instable, $M S S$ microsatellite stable, $p M M R$ proficient MMR protein expression 
Fig. 1 Comparison of the mutated biomarker spectrum between MSI CRC cases and MSI EC cases detected by the Idylla MSI test. Two-tailed unpaired $t$ test, $P<0.05$

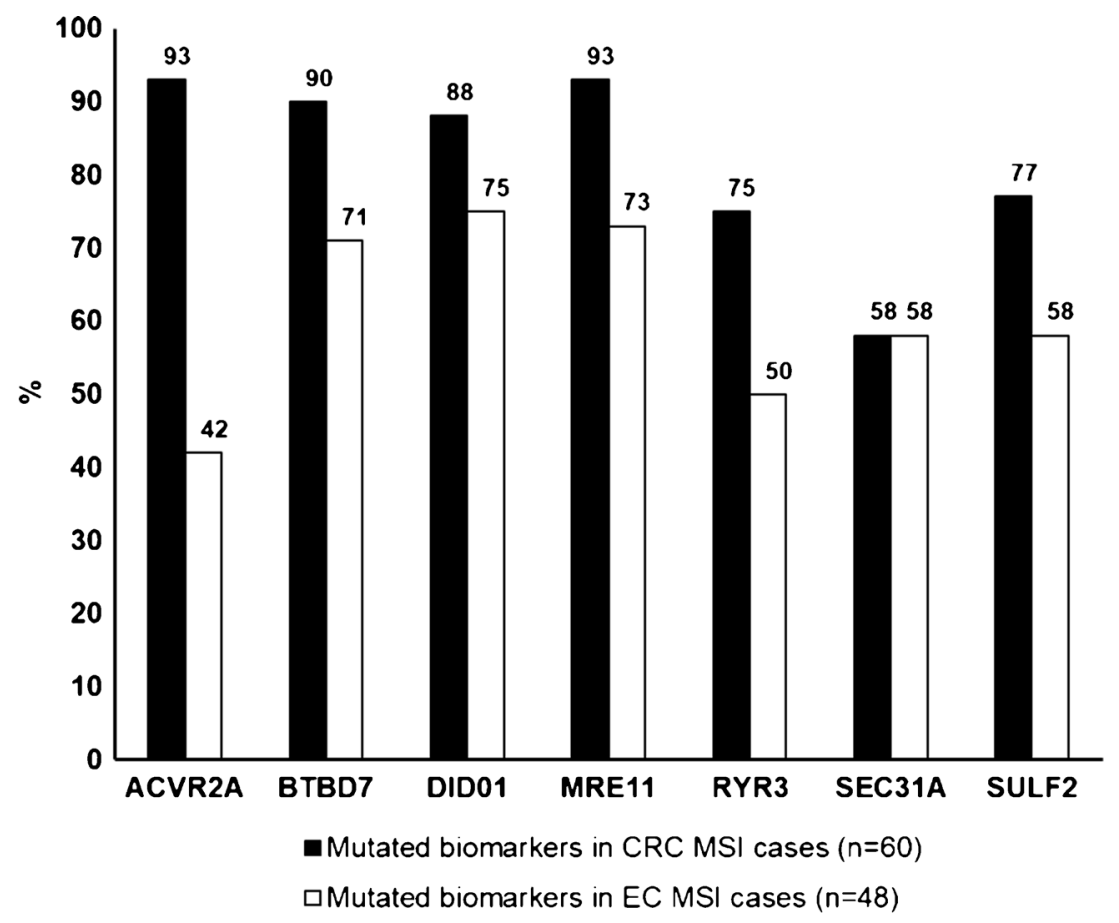

\section{Comparison between Idylla MSI test and IHC in consecutive series of endometrial cancer}

Patients of the consecutive EC sample series (Set I, $n=108$ ) had median age of 71 years (range from 34 to 90). Tumor cell percentage estimated for the Idylla analysis varied between 30 and $90 \%$. None of the Idylla test results were invalid, and 27/ 108 were MSI and 81/108 MSS. IHC, in turn, showed dMMR protein expression in 33/108 samples and pMMR protein expression in 75/108 samples. We thus identified 6/108 (5.6\%) discrepant cases between IHC (all dMMR) and the Idylla test (all MSS), the overall agreement being 94.4\% (102/108) (Table 2; two-tailed Fisher's exact test, $P<0.0001$ ). Loss of MLH1 protein (and concomitant loss of PMS2 protein) was detected in 27/33, loss of MSH2 (and concomitant loss of MSH6) protein expression in 4/33, an isolated loss of MSH6 protein expression in $1 / 33$, and combined loss of MLH1, PMS2, and MSH6 expression in 1/33 samples. In the latter case, however, the loss of MSH6 protein expression was not

Table 2 Concordance between MMR protein IHC analysis and Idylla MSI test in the consecutive EC Set I $(n=108)$

\begin{tabular}{llll}
\hline & & Idylla \\
\hline & & MSI & MSS \\
IHC & dMMR & 27 & 6 \\
& pMMR & 0 & 75 \\
\hline
\end{tabular}

$d M M R$ deficient MMR protein expression, $M M R$ mismatch repair, MSI microsatellite instable, MSS microsatellite stable, $p M M R$ proficient MMR protein expression homogeneous, which may suggest a somatic mutation in the microsatellites of MSH6 gene due to the MLH1 deficiency [13]. Using IHC analysis as a reference, sensitivity of Idylla test was $81.8 \%$ and specificity $100 \%$, while positive predictive value (PPV) was $100 \%$ and negative predictive value (NPV) $92.6 \%$. With IHC, four of these discrepant cases were in the MLH1 deficient sample group $(4 / 28,14.3 \%)$, one was in the MSH2 deficient group $(1 / 4,25 \%)$, and one was the only MSH6 deficient sample (1/1, 100\%) (Table 3).

\section{Analysis of the Idylla MSI test limit of detection for endometrial cancer samples}

Since there is no IVD-CE claim for the EC tissue samples, we next studied the limit of detection for the Idylla MSI test. For that, eight concordant dMMR IHC and Idylla MSI EC Set I samples were selected and macrodissected in order to obtain 21 samples with tumor cell percentage varying from 10 to

Table 3 Agreement for each MMR protein as determined by IHC and Idylla MSI test in the consecutive EC Set I ( $n=33)$

\begin{tabular}{lllll}
\hline & IHC dMMR & Idylla MSI & Idylla MSS & $\begin{array}{l}\text { MSI/dMMR } \\
\text { agreement }\end{array}$ \\
\hline MLH1 & 28 & 24 & 4 & $24 / 28(85.7 \%)$ \\
MSH2 & 4 & 3 & 1 & $3 / 4(75.0 \%)$ \\
MSH6 & 1 & 0 & 1 & $0 / 1(0.0 \%)$ \\
Total & 33 & 27 & 6 & $27 / 33(81.8 \%)$ \\
\hline
\end{tabular}

$d M M R$ deficient MMR protein expression, MMR mismatch repair, MSI microsatellite instable, MSS microsatellite stable 
$70 \%$. The MSI result was obtained in 2/8 (25\%) samples with tumor cell percentage $<20 \%(10-15 \%)$, in $2 / 3(66.7 \%)$ samples with tumor cell percentage $25 \%$, and in $10 / 10(100 \%)$ samples with tumor cell percentage $\geq 30 \%$ (30-70\%), suggesting that at least for these samples the $30 \%$ tumor cell cut off was optimal (Supplementary Table S1).

\section{Comparison between Idylla MSI test and IHC results in retrospective series of endometrial cancer}

To further validate Idylla MSI test in EC, we collected EC Set II ( $n=33)$ from cases with known dMMR protein IHC diagnosis. EC patients included into this retrospective dMMR EC sample series had median age of 60 years (range from 43 to 81). First, we restained the samples with the current MMR IHC protocol to verify the dMMR status. IHC showed loss of MLH1 protein (and concomitant loss of PMS2 protein) in 13, loss of MSH2 (and concomitant loss of MSH6) protein expression in 4, and an isolated loss of MSH6 protein expression in 16. Tumor cell percentage estimated for Idylla analysis varied between 30 and $90 \%$. The Idylla test scored all the results valid and they were MSI in 21/33 and MSS in 12/33 of the dMMR cases. We here identified 12/33 (36.3\%) discrepant cases between Idylla (all MSS) and IHC (all dMMR), the overall percent agreement between the tests being $63.6 \%$ (21/33). With IHC, 10 of these discrepant cases were of the MSH6 deficient sample group $(10 / 16,62.5 \%)$, two were of the MLH1 deficient sample group $(2 / 13,15.4 \%)$, and none were of the MSH2 deficient samples (0/4) (Table 4).

MSI/dMMR agreement for each dMMR protein group calculated from the combined EC Set I and EC Set II is presented in Table 5. In this combined EC series $(n=48)$, Idylla test detected three to seven mutated biomarkers in $41 / 48$ samples and two mutated biomarkers in $7 / 48$ samples, whereas one MSS sample had one mutated biomarker and the rest of them had seven wild-type biomarkers. On average, $61.0 \pm 12.5 \%$ of the seven unstable sites were mutated in the MSI EC cases (Fig. 1), which was significantly lower than that of the CRC cases $(82.1 \pm 12.9 \% ; P<0.05)$. Of the mutated markers, $A C V R 2 A$ and $R Y R 3$ were mutated in 20 and 24 samples

Table 4 Agreement for each MMR protein as determined by IHC and Idylla MSI test in the EC Set II $(n=33)$

\begin{tabular}{lllll}
\hline & IHC dMMR & Idylla MSI & Idylla MSS & $\begin{array}{l}\text { MSI/dMMR } \\
\text { agreement }\end{array}$ \\
\hline MLH1 & 13 & 11 & 2 & $11 / 13(84.6 \%)$ \\
MSH2 & 4 & 4 & 0 & $4 / 4(100 \%)$ \\
MSH6 & 16 & 6 & 10 & $6 / 16(37.5 \%)$ \\
Total & 33 & 21 & 12 & $21 / 33(63.6 \%)$ \\
\hline
\end{tabular}

$d M M R$ deficient MMR protein expression, $M M R$ mismatch repair, $M S I$ microsatellite instable
Table 5 Agreement for each MMR protein as determined by IHC and Idylla MSI test in all EC samples (EC Set I and Set II; $n=66$ )

\begin{tabular}{lllll}
\hline & IHC dMMR & Idylla MSI & Idylla MSS & $\begin{array}{l}\text { MSI/dMMR } \\
\text { agreement }\end{array}$ \\
\hline MLH1 & 41 & 35 & 6 & $35 / 41(85.4 \%)$ \\
MSH2 & 8 & 7 & 1 & $7 / 8(87.5 \%)$ \\
MSH6 & 17 & 6 & 11 & $6 / 17(35.3 \%)$ \\
Total & 66 & 48 & 18 & $48 / 66(72.7 \%)$ \\
\hline
\end{tabular}

$d M M R$ deficient MMR protein expression, $M M R$ mismatch repair, $M S I$ microsatellite instable

(41.7\% and 50.0\%), SEC31A and SULF2 in 28 samples (58.3\%), and the rest of the markers were mutated in 34 to 36 cases (70.8 to $75.0 \%)$. In combined EC Set I and Set II, mutated biomarkers detected by Idylla MSI test varied depending on the MMR protein deficiency. Especially this applied to the MSH6 deficient cases, which less frequently showed ACVR2A and BTBD7 mutations (both $16.7 \%$ ), but most often had mutated DIDO01 (83.3\%) (Fig. 2a). In MSH6 deficient CRC samples, similar divergences were not observed (Fig. 2b).

\section{Analysis of the discrepant endometrial cancer cases using microsatellite markers BAT25 and BAT26}

In the EC Set I, we identified six and in EC Set II 12 discrepant cases demonstrating dMMR IHC result and MSS using the Idylla test, which were first re-evaluated (tumor cell percentage) and retested with the Idylla MSI test using new paraffin slices. The result of re-testing was MSS in all 18 cases. We also re-evaluated IHC staining patterns and identified one case (B107) with heterogeneous loss of MLH1 (and concomitant PMS2) (Supplementary Fig. S1). Subclonal loss of MMR protein expression and heterogeneous dMMR IHC staining has been reported to occur in up to $7 \%$ of ECs, and exclusively in glandular endometrioid component [14], as in the case of B107. It is also noteworthy that this heterogeneous loss of MLH1 is suggestive for sporadic rather than germline deficiency. As a third method to evaluate the MSI phenotype, the discrepant cases (from EC Set I to Set II) were further tested for RER status using BAT25 and BAT26 microsatellite markers (Table 6). For this analysis, two samples (B14 and C26) did not meet the required 50\% tumor cell content [12], and two (B107 and C9) did not have enough tissue available. Of the 14 samples that were subjected to RER analysis, four were shown to be positive for both BAT25 and BAT26, indicating MSI, and rest of them were negative (Table 6). These four RER positive cases had been shown to be deficient for MSH6 protein expression. 
Fig. 2 Mutated biomarker spectrums for each dMMR IHC protein in MSI EC and CRC cases detected by the Idylla MSI test. a Combined MSI cases from EC

Sets I and II $(n=48)$ and $\mathbf{b}$ combined MSI cases from CRC Sets I and II $(n=60)$
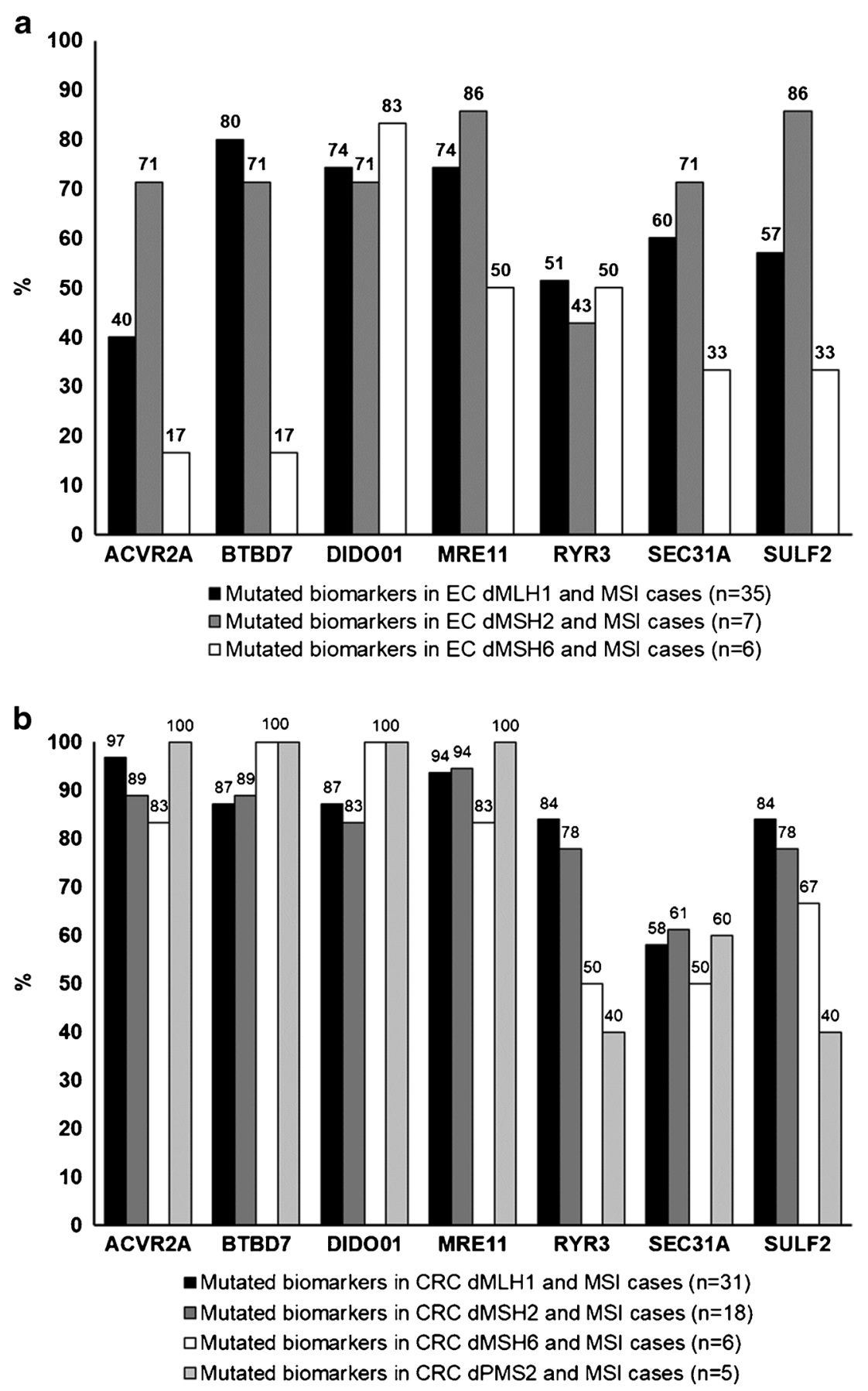

\section{Discussion}

In this study, we evaluated the diagnostic performance of Idylla MSI test in detecting MSI in CRC $(n=128)$ and EC $(n=141)$ samples. The Idylla test showed $100 \%$ concordance with the MMR IHC results in the CRC cohort but only $87.2 \%$ concordance in the EC cohort. None of the Idylla analysis failed due to technical errors or sample-related problems. Strength of the study includes real-life prospective blinded design of the 100 consecutive CRC cases. We were not able to perform identical study protocol for the EC cases, which were analyzed in two cohorts, i.e., a consecutive and blinded Set I and a separate historical Set II with known dMMR IHC status. As compared to IHC, sensitivity of the Idylla test was $72.7 \%$ and specificity $100 \%$ in EC samples. Eighteen discrepant EC cases between IHC and Idylla test were detected, and we were able to subject 14 of these to RER analysis using two mononucleotide markers BAT25 and BAT26. Four cases were identified that were repeatedly MSS in the Idylla analysis but were found to be MSI when using the BAT25 and BAT26 
Table 6 Characteristics of the 18 discrepant (Idylla MSS versus IHC dMMR) EC cases

\begin{tabular}{|c|c|c|c|c|c|c|c|c|}
\hline Case & $\begin{array}{l}\text { Age } \\
\text { (years) }\end{array}$ & $\begin{array}{l}\text { Tumor } \\
\text { cells } \\
(\%)\end{array}$ & $\begin{array}{l}\text { Tissue } \\
\text { area } \\
\left(\mathrm{mm}^{2}\right)\end{array}$ & $\begin{array}{l}\text { Macro- } \\
\text { dissection }\end{array}$ & $\begin{array}{l}\text { IHC (loss } \\
\text { of MMR } \\
\text { proteins) }\end{array}$ & $\begin{array}{l}\text { Idylla } \\
\text { MSI } \\
\text { analysis }\end{array}$ & $\begin{array}{l}\text { Number of } \\
\text { mutated } \\
\text { biomarkers }\end{array}$ & $\begin{array}{l}\text { RER analysis } \\
\text { (BAT25 and } \\
\text { BAT26) }\end{array}$ \\
\hline B14 & 69 & 30 & 300 & Yes & MSH2 & MSS & $0 / 7$ & $\mathrm{ND}^{\mathrm{a}}$ \\
\hline B66 & 79 & 50 & 40 & Yes & MLH1 & MSS & $0 / 7$ & Negative \\
\hline B79 & 72 & 50 & 30 & Yes & MSH6 & MSS & $0 / 7$ & Negative \\
\hline B80 & 73 & 40 & 50 & Yes & MLH1 & MSS & $0 / 7$ & Negative \\
\hline B97 & 53 & 60 & 75 & Yes & MLH1 & MSS & $0 / 7$ & Negative \\
\hline B107 & 77 & 70 & 100 & Yes & MLH1 & MSS & $0 / 7$ & $\mathrm{ND}^{\mathrm{b}}$ \\
\hline $\mathrm{C} 1$ & 59 & 40 & 50 & Yes & MSH6 & MSS & $0 / 7$ & Negative \\
\hline $\mathrm{C} 4$ & 72 & 60 & 50 & Yes & MSH6 & MSS & $0 / 7$ & Negative \\
\hline $\mathrm{C} 7$ & 58 & 50 & 300 & No & MSH6 & MSS & $0 / 7$ & Positive \\
\hline C9 & 66 & 70 & 15 & Yes & MSH6 & MSS & $0 / 7$ & $\mathrm{ND}^{\mathrm{b}}$ \\
\hline $\mathrm{C} 13$ & 63 & 80 & 75 & Yes & MSH6 & MSS & $0 / 7$ & Negative \\
\hline $\mathrm{C} 17$ & 78 & 80 & 75 & Yes & MSH6 & MSS & $0 / 7$ & Positive \\
\hline C19 & 69 & 40 & 100 & Yes & MLH1 & MSS & $0 / 7$ & Negative \\
\hline $\mathrm{C} 20$ & 61 & 80 & 150 & No & MSH6 & MSS & $0 / 7$ & Negative \\
\hline $\mathrm{C} 21$ & 49 & 80 & 75 & Yes & MSH6 & MSS & $0 / 7$ & Positive \\
\hline $\mathrm{C} 22$ & 72 & 80 & 100 & Yes & MLH1 & MSS & $1 / 7$ & Negative \\
\hline $\mathrm{C} 26$ & 80 & 30 & 25 & Yes & MSH6 & MSS & $0 / 7$ & $\mathrm{ND}^{\mathrm{a}}$ \\
\hline $\mathrm{C} 29$ & 58 & 60 & 100 & Yes & MSH6 & MSS & $0 / 7$ & Positive \\
\hline
\end{tabular}

dMMR deficient MMR protein expression, IHC immunohistochemistry, MMR mismatch repair, MSI microsatellite instable, MSS microsatellite stable, $N D$ not determined, $R E R$ replication error

${ }^{\mathrm{a}}$ Tumor cell percentage less than $50 \%$

${ }^{\mathrm{b}}$ Not enough tissue available markers. All these cases were deficient for expression of MSH6 protein. Weakness of this study was that we used only two mononucleotide microsatellite markers instead of five, which may have been more sensitive in detecting MSI [6].

One explanation for discrepancies between IHC and DNAbased assay could depend on the amount of tumor cells included to the FFPE flakes. To this end, we scrutinized the minimum tumor cellularity requirement for Idylla MSI test in the EC samples. Our data suggest that at least 30\% EC cell percentage is required for the Idylla analysis. Further, low tumor cell number does not explain the discrepant cases that we found between IHC and the Idylla test, since all four RERpositive (BAT25 and BAT26 positive) cases had tumor cell percentages between 50 and $80 \%$. It should be pointed out that we also included MSH6 losses to the CRC cohort $(n=6)$ that were all correctly identified as MSI using the Idylla test. Our results are supported by previous findings showing that MSI in EC demonstrates a higher frequency of minimal (1-2 nucleotide) microsatellite shifts especially in the case of MSH6 loss, which is a challenge for DNA-based MSI assays [9, 15, 16]. Importantly, detection of MSH6 deficiency is in parts crucial, since females carrying a pathological MSH6 mutation are reported to be at especially high risk of endometrial cancer compared with other LS-related cancers [17].
Previous reports have also found a very high concordance (97.6-100\%; $n=42-105)$ of the Idylla MSI test with reference tests in retrospective CRC samples [18-20]. In a larger sample set, Zwaenepoel et al. reported the Idylla test to show $98.7 \%$ overall agreement using historical and partially dMMRenriched IHC data in 330 CRC samples [21]. In this report, one case was MSS in Idylla analysis, whereas IHC was dMMR and Promega MSI analysis was MSI and interestingly tumor mutation burden was very high, whereas three IHC dMMR cases showed MSS in DNA-based assays and low tumor mutation burden. In addition, a multi-center real-life global study including 44 clinical centers and 1301 CRC samples showed the concordance level between the Idylla test and IHC to be as high as $96.4 \%$, with Idylla having lower failure rates [11].

Excellent accuracy of the Idylla MSI test in CRC samples has encouraged to study the diagnostic performance of this novel assay in other solid tumors as well. Farmkiss et al. compared retrospectively IHC and the Idylla test across 50 biopsies of gastric adenocarcinoma, scoring concordant results in 48 samples with a $95.7 \%$ sensitivity and $100 \%$ specificity [22]. Pécriaux et al. have evaluated the Idylla test in a panel of solid tumors, including $15 \mathrm{EC}$ samples using extracted DNA, and reported sensitivity of $89 \%$ and specificity of $100 \%$ to detect MSI status in EC samples as compared to IHC [23]. It is important to note 
that all nine MSI cases in this report were MLH1 deficient. Gilson et al. reported similarly with $15 \mathrm{EC}$ samples a $100 \%$ specificity and sensitivity with the Idylla test as compared to previously used PCR-based Promega MSI analysis, but if compared to IHC, the sensitivity seems to be $80-90 \%$ and specificity $100 \%$ [24]. Our study agrees with these reports on the excellent specificity of the Idylla MSI test in EC diagnostics, but the sensitivity when compared to IHC was clearly lower in our study $(72.7 \%)$, which to our knowledge is the largest EC study thus far $(n=141)$.

Our study showed significant differences in the mutated biomarker spectrum between CRC and EC MSI cases detected by the Idylla MSI test. First, on average $82.1 \%$ of the seven unstable sites were mutated in MSI CRC, whereas only $61.0 \%$ of the sites were mutated in MSI EC cases. This is in line with the previous publication reporting lower proportion of unstable markers per tumor in $\mathrm{EC}$ as compared to $\mathrm{CRC}$, even in the tumors originating from the same LS patient [25]. Second, our results implicate that mutated biomarkers $A C V R 2 A$ and RYR3 are more frequently mutated in CRC when compared to EC, whereas SEC31A seems to be mutated by the same frequency in both CRC and EC MSI samples. Previously, Kim et al. have showed that $A C V R 2 A$ is indeed a gene harboring frameshift microsatellite instability specifically in CRC and less often in EC genomes [26]. They also demonstrated SEC31A to be a gene with no extreme specificity to harbor frameshift MSI in either CRC or EC genomes. Further, the multicenter Idylla MSI test study of CRC reported ACVR2A to be the most often mutated biomarker $(94.0 \%)$ and SEC 31A to be the least mutated marker (60.9\%) [11], and our frequencies are in line with a sequencing study demonstrating ACVR2A mutations in $92 \%$ and SEC $31 A$ mutations in $54 \%$ of one hundred MSI CRC cases [27].

In conclusion, our study reinforces the accuracy of the fast and automated Idylla MSI test to detect MSI phenotype in CRC samples. In EC samples, Idylla test is $100 \%$ specific, but sensitivity is compromised especially in the case of MSH6 deficient tumors. We conclude that the Idylla test offers a sensitive and specific method for CRC diagnostics, but it should be validated for each tumor type separately in a relatively large material before applying it to diagnostic use.

\begin{abstract}
Abbreviations CRC, Colorectal cancer; dMMR, Deficient MMR; EC, Endometrial cancer; FFPE, Formalin-fixed paraffin-embedded; HUH, Helsinki University Hospital; IHC, Immunohistochemistry; LS, Lynch syndrome; MMR, Mismatch repair; MSI, Microsatellite instability; MSS, Microsatellite stable; NGS, Next-generation sequencing; NPV, Negative predictive value; pMMR, Proficient MMR; PPV, Positive predictive value; RER, Replication error
\end{abstract}

Supplementary Information The online version contains supplementary material available at https://doi.org/10.1007/s00428-021-03082-w.

Acknowledgements We thank Merja Haukka and Carita Liikanen for excellent technical assistance and Biocartis for providing the Idylla platform and cartridges.
Code availability Not applicable.

Author contribution All authors contributed to the study conception and design. Material preparation, data collection, and analysis were performed by Iiris Ukkola, Pirjo Nummela, Annukka Pasanen, Soili Kytölä, Ralf Bützow, and Ari Ristimäki. The first draft of the manuscript was written by Iiris Ukkola, Pirjo Nummela, and Ari Ristimäki, and all authors commented on previous versions of the manuscript. All authors read and approved the final manuscript.

Funding Open access funding provided by University of Helsinki including Helsinki University Central Hospital. Biocartis provided the Idylla platform and cartridges. This study was funded by Finnish Cancer Organizations, Finska Läkaresällskapet, Helsinki University Central Hospital Research Funds, Sigrid Juselius Foundation, and University of Helsinki. The funding sources or Biocartis had no role in study design, data collection and analysis, decision to publish, or preparation of the manuscript.

Data availability The data obtained during the current study are available from the corresponding author AR on reasonable request.

\section{Declarations}

Ethics approval This study was approved by the Ethics Committee of the Helsinki University Central Hospital, and all methods were performed in accordance with the relevant guidelines and regulations. Data were anonymized prior to use for the study.

Consent to participate Not applicable.

Consent for publication Not applicable.

Competing interests The authors declare no competing interests.

Open Access This article is licensed under a Creative Commons Attribution 4.0 International License, which permits use, sharing, adaptation, distribution and reproduction in any medium or format, as long as you give appropriate credit to the original author(s) and the source, provide a link to the Creative Commons licence, and indicate if changes were made. The images or other third party material in this article are included in the article's Creative Commons licence, unless indicated otherwise in a credit line to the material. If material is not included in the article's Creative Commons licence and your intended use is not permitted by statutory regulation or exceeds the permitted use, you will need to obtain permission directly from the copyright holder. To view a copy of this licence, visit http://creativecommons.org/licenses/by/4.0/.

\section{References}

1. Yamamoto H, Imai K (2015) Microsatellite instability: an update. Arch Toxicol 89:899-921

2. Diao Z, Han Y, Chen Y, Zhang R, Li J (2021) The clinical utility of microsatellite instability in colorectal cancer. Crit Rev Oncol/ Hematol 157:103171

3. Hause RJ, Pritchard CC, Shendure J, Salipante SJ (2016) Classification and characterization of microsatellite instability across 18 cancer types. Nat Med 22:1342-1350

4. Gupta R, Sinha S, Paul RN (2018) The impact of microsatellite stability status in colorectal cancer. Curr Probl Cancer 42:548-559 
5. Deshpande M, Romanski PA, Rosenwaks Z, Gerhardt J (2020) Gynecological cancers caused by deficient mismatch repair and microsatellite instability. Cancers 12:3319

6. Luchini C, Bibeau F, Ligtenberg MJL, Singh N, Nottegar A, Bosse T, Miller R, Riaz N, Douillard J, Andre F, Scarpa A (2019) ESMO recommendations on microsatellite instability testing for immunotherapy in cancer, and its relationship with PD-1/PD-L1 expression and tumour mutational burden: a systematic review-based approach. Ann Oncol 30:1232-1243

7. Gordhandas S, Kahn RM, Gamble C, Talukdar N, Maddy B, Baltich Nelson B, Askin G, Christos PJ, Holcomb K, Caputo TA, Chapman-Davis E, Frey MK (2020) Clinicopathologic features of endometrial cancer with mismatch repair deficiency. Ecancermedicalscience 14:1061

8. Seppälä TT, Latchford A, Negoi I, Soares AS, Jimenez-Rodriguez R, Sánchez-Guillén L, Evans DG, Ryan N, Crosbie EJ, Dominguez-Valentin M, Burn J, Kloor M, Von Knebel DM, Van Duijnhoven FJB, Quirke P, Sampson JR, Møller P (2020) European guidelines from the EHTG and ESCP for Lynch syndrome: an updated third edition of the Mallorca guidelines based on gene and gender. Br J Surg. https://doi.org/10.1002/bjs.11902

9. Palomäki GE, McClain MR, Melillo S, Hampel HL, Thibodeau SN (2009) EGAPP supplementary evidence review: DNA testing strategies aimed at reducing morbidity and mortality from Lynch syndrome. Genet Med 11:42-65

10. Stinton C, Fraser H, Al-Khudairy L, Court R, Jordan M, Grammatopoulos D, Taylor-Phillips S (2021) Testing for lynch syndrome in people with endometrial cancer using immunohistochemistry and microsatellite instability-based testing strategies - a systematic review of test accuracy. Gynecol Oncol 160:148-160

11. Velasco A, Tokat F, Bonde J, Trim N, Bauer E, Meeney A, de Leng W, Chong G, Dalstein V, Kis LL, Lorentzen JA, Tomić S, Thwaites K, Putzová M, Birnbaum A, Qazi R, Primmer V, Dockhorn-Dworniczak B, Hernández-Losa J, Soares FA, Gertler AA, Kalman M, Wong C, Carraro DM, Sousa AC, Reis RM, Fox SB, Fassan M, Brevet M, Merkelbach-Bruse S, Colling R, Soilleux E, Teo RYW, D'Haene N, Nolet S, Ristimäki A, Väisänen T, Chapusot C, Soruri A, Unger T, Wecgowiec J, Biscuola M, Frattini M, Long A, Campregher PV, Matias-Guiu X (2020) Multi-center real-world comparison of the fully automated Idylla ${ }^{\mathrm{TM}}$ microsatellite instability assay with routine molecular methods and immunohistochemistry on formalin-fixed paraffinembedded tissue of colorectal cancer. Virchows Arch. https://doi.org/ 10.1007/s00428-020-02962-x

12. Loukola A, Eklin K, Laiho P, Salovaara R, Kristo P, Järvinen H, Mecklin JP, Launonen V, Aaltonen LA (2001) Microsatellite marker analysis in screening for hereditary nonpolyposis colorectal cancer (HNPCC). Cancer Res 61:4545-4549

13. Shia J, Zhang L, Shike M, Guo M, Stadler Z, Xiong X, Tang LH, Vakiani E, Katabi N, Wang H, Bacares R, Ruggeri J, Boland CR, Ladanyi M, Klimstra DS (2013) Secondary mutation in a coding mononucleotide tract in MSH6 causes loss of immunoexpression of MSH6 in colorectal carcinomas with MLH1/PMS2 deficiency. Mod Pathol 26:131-138

14. Watkins JC, Nucci MR, Ritterhouse LL, Howitt BE, Sholl LM (2016) Unusual mismatch repair immunohistochemical patterns in endometrial carcinoma. Am J Surg Pathol 40:909-916

15. Wang Y, Shi C, Eisenberg R, Vnencak-Jones CL (2017) Differences in microsatellite instability profiles between endometrioid and colorectal cancers: a potential cause for falsenegative results? J Mol Diagn 19:57-64

16. Wu X, Snir O, Rottmann D, Wong S, Buza N, Hui P (2019) Minimal microsatellite shift in microsatellite instability high endometrial cancer: a significant pitfall in diagnostic interpretation. Mod Pathol 32:650-658

17. Dominguez-Valentin M, Sampson JR, Seppälä TT, ten Broeke SW, Plazzer JP, Nakken S, Engel C, Aretz S, Jenkins MA, Sunde L,
Bernstein I, Capella G, Balaguer F, Thomas H, Evans DG, Burn J, Greenblatt M, Hovig E, de Vos tot Nederveen Cappel WH, Sijmons RH, Bertario L, Tibiletti MG, Cavestro GM, Lindblom A, Della Valle A, Lopez-Köstner F, Gluck N, Katz LH, Heinimann K, Vaccaro CA, Büttner R, Görgens H, Holinski-Feder E, Morak M, Holzapfel S, Hüneburg R, Knebel Doeberitz M, Loeffler M, Rahner N, Schackert HK, Steinke-Lange V, Schmiegel W, Vangala D, Pylvänäinen K, Renkonen-Sinisalo L, Hopper JL, Win AK, Haile RW, Lindor NM, Gallinger S, le Marchand L, Newcomb PA, Figueiredo JC, Thibodeau SN, Wadt K, Therkildsen C, Okkels H, Ketabi Z, Moreira L, Sánchez A, Serra-Burriel M, Pineda M, Navarro M, Blanco I, Green K, Lalloo F, Crosbie EJ, Hill J, Denton OG, Frayling IM, Rødland EA, Vasen H, Mints M, Neffa F, Esperon P, Alvarez K, Kariv R, Rosner G, Pinero TA, Gonzalez ML, Kalfayan P, Tjandra D, Winship IM, Macrae F, Möslein G, Mecklin JP, Nielsen M, Møller P (2020) Cancer risks by gene, age, and gender in 6350 carriers of pathogenic mismatch repair variants: findings from the Prospective Lynch Syndrome Database. Genet Med 22:15-25

18. Lee M, Chun S, Sung CO, Kim SY, Kim TW, Jang SJ, Kim J (2019) Clinical utility of a fully automated microsatellite instability test with minimal hands-on time. J Pathol Transl Med 53:386-392

19. Li X, Xu J, Li L, Mu X, Wang Y, Li X (2019) Evaluation of a fully automated Idylla test system for microsatellite instability in colorectal cancer. Clin Colorectal Cancer 18:e316-e323

20. Mindiola-Romero AE, Green DC, Al-Turkmani MR, Godwin KN, Mackay AC, Tafe LJ, Ren B, Tsongalis GJ (2020) Novel Biocartis Idylla $^{\mathrm{TM}}$ cartridge-based assay for detection of microsatellite instability in colorectal cancer tissues. Exp Mol Pathol 116:104519

21. Zwaenepoel K, Holmgaard Duelund J, De Winne K, Maes V, Weyn C, Lambin S, Dendooven R, Broeckx G, Steiniche T, Pauwels P (2019) Clinical performance of the Idylla MSI test for a rapid assessment of the DNA microsatellite status in human colorectal cancer. J Mol Diagn 22:386-395

22. Farmkiss L, Hopkins I, Jones M (2020) Idylla microsatellite instability assay versus mismatch repair immunohistochemistry: a retrospective comparison in gastric adenocarcinoma. J Clin Pathol. https://doi.org/10.1136/jclinpath-2020-207033

23. Pécriaux A, Favre L, Calderaro J, Charpy C, Derman J, Pujals A (2020) Detection of microsatellite instability in a panel of solid tumours with the Idylla MSI Test using extracted DNA. J Clin Pathol 74:36-42

24. Gilson P, Levy J, Rouyer M, Demange J, Husson M, Bonnet C, Salleron J, Leroux A, Merlin JL, Harlé A (2020) Evaluation of 3 molecular based assays for microsatellite instability detection in formalin fixed tissues of patients with endometrial and colorectal cancers. Sci Rep 10:16386

25. Kuismanen SA, Moisio A, Schweizer P, Truninger K, Salovaara R, Arola J, Butzow R, Jiricny J, Nyström-Lahti M, Peltomäki P (2002) Endometrial and colorectal tumors from patients with hereditary nonpolyposis colon cancer display different patterns of microsatellite instability. Am J Pathol 160:1953-1958

26. Kim T, Laird P, Park P (2013) The landscape of microsatellite instability in colorectal and endometrial cancer genomes. Cell 155:858-868

27. Alhopuro P, Sammalkorpi H, Niittymäki I, Biström M, Raitila A, Saharinen J, Nousiainen K, Lehtonen HJ, Heliövaara E, Puhakka J, Tuupanen S, Sousa S, Seruca R, Ferreira AM, Hofstra RMW, Mecklin J, Järvinen H, Ristimäki A, Ørntoft TF, Hautaniemi S, Arango D, Karhu A, Aaltonen LA (2012) Candidate driver genes in microsatellite-unstable colorectal cancer. Int J Cancer 130:1558-1566

Publisher's note Springer Nature remains neutral with regard to jurisdictional claims in published maps and institutional affiliations. 